transmembrane helices leading to an extracellular $\mathrm{N}$-terminus and an extracellular loop between helices two and three. The C242T polymorphism causes a change of His72 to Tyr72. This change results in significant morphological changes of the extracellular loop of the p22phox, which is in the putative interactive region of the p22phox with the catalytic subunit (Nox2). This may interfere with their association, and subsequently result in a reduced cytochrome $b$ function and a reduced ROS production by NADPH oxidase. These results give us insight into the molecular mechanism of this polymorphism in reducing vascular oxidative stress and may explain how this polymorphism is linked with reduced incidence of CHD.

\section{BAS/ HEPATOCYTE GROWTH FACTOR/C-MET SIGNALLING BSCR49 ACTIVATES NOTCH TRANSLOCATION AND IS ASSOCIATED WITH SMOOTH MUSCLE CELL MINERALISATION IN VITRO}

doi:10.1136/hrt.2010.205781.60

J Durrand, Y Liu, T Wang, M Y Alexander. Cardiovascular Research Group, Faculty of Medicine \& Human Science, School of Biomedicine, University of Manchester, UK

Vascular calcification is an established pathological process that contributes to several forms of cardiovascular morbidity-notably, atherosclerosis. The molecular mechanisms involved continue to be explored. Hepatocyte growth factor (HGF) signalling, via its receptor c-MET, has been identified in association with atherosclerotic plaque development. We have demonstrated that overexpression of HGF in human smooth muscle cells (hSMC) accelerates their mineralisation. Reports demonstrating upregulation of the notch ligand $\delta$, and the presence of a feedback loop linked to the c-MET pathway, raise the possibility that the effects of HGF on mineralisation may be mediated via notch signalling. We aim to test the hypothesis that notch signalling is involved in Ad-HGFinduced in vitro mineralisation of hSMCs. We demonstrate accelerated mineralisation in response to adenoviral-mediated HGF overexpression, confirmed by alizarin red staining, calcium incorporation and increased alkaline phosphatase activity. In addition, we show upregulation and phosphorylation of c-MET and reduction of the mineralisation inhibitor osteopontin. We identify upregulation of the notch-3 intracellular domain via western blot analysis and, using immunocytochemistry, show an altered distribution of notch3 in Ad-HGF-infected cells. Finally, we show (i) an attenuation of mineralisation in hSMCs following overexpression of NK4, the cMET antagonist and (ii) that treatment of hSMCs with DAPT, the notch inhibitor, also decreased the rate of mineralisation compared with Ad-HGF infected cells and controls. These findings suggest a link with the notch pathway as a possible downstream effector of HGF and further elucidate a novel mechanism underpinning vascular calcification.

\section{BAS/ EFFECT OF IMMUNISATION WITH CHLAMYDIA BSCR50 PNEUMONIAE RECOMBINANT MAJOR OUTER MEMBRANE PROTEIN ON ATHEROSCLEROSIS DEVELOPMENT}

doi:10.1136/hrt.2010.205781.61

${ }^{1} \mathrm{R}$ El Kadri, ${ }^{1} \mathrm{~A}$ Bermudez-Fajardo, ${ }^{2} \mathrm{M}$ Puolakkainen, ${ }^{1} \mathrm{G}$ Stewart, ${ }^{1} \mathrm{E}$ Oviedo-Orta. ${ }^{1}$ University of Surrey, Faculty of Health and Medical Sciences, Guildford, Surrey, UK; ${ }^{2}$ Department of Viral Diseases and Immunology, NPHI, Helsinki, Finland

The major outer membrane protein (MOMP) of Chlamydia pneumoniae is a $40 \mathrm{kDa}$ porin and represents approximately $60 \%$ of the outer membrane protein pool. Previous studies have shown that MOMP can dampen down T-cell-mediated immune responses. We decided to assess if this effect could have an impact on atherosclerotic plaque development. We used recombinant Mycobacterium vaccae ( $M$ vaccae) vectors expressing MOMP (with and without signal sequence) to vaccinate ApoE-/- mice intranasally. Animals received one initial dose and two booster doses 3 weeks apart and continued with a standard Chow diet for another 4 weeks. Control mice received phosphate-buffered saline or were left untreated. Plasma collected before immunisation and at termination was used to measure levels of interferon $\gamma$, interleukin (IL)-4 and IL-10 and also IgG1 and IgG2b. Atherosclerotic plaque development was assessed using paraffin sections of the brachiocephalic artery. Our results show that intranasal administration of $M$ vaccae vectors expressing MOMP, with or without signal sequence, promotes antiatherogenic T-cell responses and halts the development and progression of atherosclerotic plaques in $\mathrm{ApoE}-/-$ mice.

\section{BAS/ CONTROL OF VASCULAR CELL INFLAMMATORY BSCR51 RESPONSES THROUGH TILRR, AN INTERLEUKIN 1 CO-RECEPTOR}

doi:10.1136/hrt.2010.205781.62

Monica Neilan, Xiao Zhang, Sheila Francis, Eva E Owarnstrom. Cell Biology, Vascular Science, University of Sheffield, UK

Inflammatory responses are induced by members of the Toll-Like and interleukin (IL)-1 receptor family and controlled by NF- $\mathrm{KB}$.

We have identified an IL-1RI co-receptor, TILRR, which potentiates activation of NF- $\kappa B$ and inflammatory responses. We show that induction of amplification depends on formation of a TILRRcontaining receptor complex, which imparts enhanced recruitment of the MyD88 adaptor to the signalling receptor IL-1RI, and induction of Ras-dependent amplification of NF- $\kappa \mathrm{B} .{ }^{1}$

We have confirmed expression of TILRR in vascular cells and have demonstrated a correlation of the level of TILRR expression with the level of NF- $\mathrm{KB}$ activity and inflammatory responses, induced by IL-1 stimulation and by mechanotransduction.

Our recent studies have demonstrated expression of TILRR in vascular endothelial cells using immunohistochemistry. Sections of perfusion-fixed, paraffin-embedded vascular tissue were stained using a specific rabbit polyclonal anti-TILRR antibody, followed by incubation with a biotinylated goat anti-rabbit antibody. Ongoing studies using wild type and ApoE-/- mice are designed to assess the impact of diet on TILRR expression and on its association with the signalling partner IL-1RI.

Acknowledgements Studies were supported by BBSRC and the BHF.

\section{REFERENCE}

1. Zhang $\mathbf{X}$, Shephard F, Kim HB, et al. TILRR-A novel a novel IL-1RI- co-receptor potentiates MyD88-recruitment to control Ras-dependent amplification of NF- $\mathrm{KB}$. J Biol Chem 2010:285:7222-32.

\section{BAS/ INVESTIGATING THE IMPORTANCE OF HEPARAN SULPHATE BSCR52 IN DETERMINING ENDOTHELIAL PROGENITOR CELL FUNCTION}

doi:10.1136/hrt.2010.205781.63

${ }^{1} \mathrm{~K}$ Williamson, ${ }^{2} \mathrm{~S}$ Haque, ${ }^{2} \mathrm{~N}$ B Bruce, ${ }^{1} \mathrm{~S}$ E Stringer, ${ }^{1} \mathrm{M}$ Y Alexander. ${ }^{1}$ Cardiovascular Research Group, University of Manchester, Manchester, UK; ${ }^{2}$ ARC Epidemiology Unit, University of Manchester, Manchester, UK; ${ }^{3}$ University of Manchester. University of Manchester, Manchester, UK

The increased risk of cardiovascular disease with age may, in part, be due to a decline in the function of endothelial progenitor cells (EPCs). Cell surface heparan sulphate proteoglycans (HSPGs) can 